\title{
The effect of olfactory training on olfactory bulb volumes in patients with idiopathic olfactory loss*
}

\author{
Mehmet K. Mahmut ${ }^{\sharp 1,2}$, Martina Musch", ${ }^{\#}$, Pengfei Han 1,3,4, Nasreddin \\ Abolmaali ${ }^{5}$, Thomas Hummel ${ }^{1}$ \\ Rhinology 58: 4, 413 - 415, 2020 \\ https://doi.org/10.4193/Rhin20.223 \\ 'Smell and Taste Clinic, Department of Otorhinolaryngology, TU Dresden, Dresden, Germany \\ *Received for publication: \\ 2 Food, Flavour and Fragrance Lab, Department of Psychology, Macquarie University, Sydney, Australia \\ May 6, 2020 \\ ${ }^{3}$ Faculty of Psychology, Southwest University, Chongqing, China \\ Accepted: May 29, 2020 \\ ${ }^{4}$ Key Laboratory of Cognition and Personality, Ministry of Education, Southwest University, Chongqing, China \\ ${ }^{5}$ Department of Radiology, Municipal Hospital and Academic Teaching Hospital of the Technical University Dresden, Dresden- \\ "Contributed equally \\ Friedrichstadt, Dresden, Germany
}

\section{To the Editor:}

Olfactory loss impacts around $20 \%$ of the population and is associated with the reduction of pleasure from eating and drinking, sex and depression ${ }^{(1)}$. Encouragingly, research findings have consistently demonstrated that olfactory training (OT) can improve olfactory function in people with olfactory loss due to various aetiologies ${ }^{(2)}$. The most commonly used method for OT involves smelling four different odours (lemon, eucalyptus, rose and cloves), twice daily, for 12 weeks.

Significant research attention has also been focussed on olfactory bulb $(\mathrm{OB})$ volume of patients with olfactory loss, because of their important role in the olfactory processing pathway ${ }^{(3)}$ and that larger $\mathrm{OB}$ volume is associated with better olfactory function ${ }^{(4)}$. Moreover, the neuroplasticity of the $\mathrm{OBs}{ }^{(3)}$ suggests that olfactory function may improve with $\mathrm{OB}$ regeneration and OT may enhance OB volume. However, whether OT will increase $O B$ volumes in patients with idiopathic olfactory loss remains unknown. Therefore, the aim of the current, two-part study was to first investigate whether patients with idiopathic olfactory loss differed from controls in terms of their OB volume and second, to investigate whether OT will enhance OB volumes in idiopathic patients.

\section{Materials and methods}

\section{Participants}

Fifty-four participants completed Part 1 of the study. Twenty-seven were patients ( 12 males, 15 females), aged $46-81$ years ( $M$ $=66.1, \mathrm{SD}=10.1)$ with idiopathic olfactory loss for an average of 4.4 years ( $S D=4.5)$. Twenty-seven participants with the same sex distribution ( 12 males, 15 females) and very similar age range ( 50 - 82 years) and mean age $(M=65.3, S D=10.1)$ formed the control group. Only the 27 patients with idiopathic olfactory loss completed Part 2. The Ethics Committee at the Medical
Faculty of the TU Dresden approved the study. All participants gave informed, written consent. Further details are provided in the supplementary section.

\section{Eligibility criteria}

Participants were interviewed in Part 1 regarding the nature and duration of their olfactory dysfunction using a medical history that included a nasal endoscopy. Idiopathic olfactory loss was diagnosed if a patient had no history of brain injury, olfactory loss did not coincide with an upper respiratory infection, medication usage, or environmental factors, neurological evaluations revealed no indications of neurodegenerative or neurovascular diseases.

\section{Procedure}

A two-part, prospective cohort study was conducted. In Part 1, each participant had a health assessment, an MRI of their OBs, and an assessment of the olfactory function using the Sniffin' Sticks. In Part 1, the OB volume and olfactory function of idiopathic patients $(\mathrm{N}=27)$ and controls $(\mathrm{N}=27)$ was compared. After a minimum of six months OT, patients (only) returned to complete Part 2 which involved a second MRI of their OB volumes, olfactory function assessment and a self-assessment of their olfactory function.

\section{Sniffin' Sticks: olfactory testing}

Olfactory function was tested in Part 1 and Part 2 using the "Sniffin' Sticks". Four variables were created based on the Sniffin' Sticks test performance: odour threshold, odour discrimination, odour identification and a combined score of all three sub-tests., referred to as the TDI (Threshold Discrimination Identification) score. 
Table 1. Descriptive statistics and statistical comparisons of control and patients in Part 1 and 2.

\begin{tabular}{|c|c|c|c|}
\hline \multirow[t]{2}{*}{ Olfactory Test Type } & \multicolumn{2}{|c|}{ Part 1 (Pre-OT) } & \multirow{2}{*}{$\begin{array}{c}\text { Part } 2 \text { (Post-OT) } \\
\text { Patients ( } \mathbf{N}=27 \text { ) Mean (SD) }\end{array}$} \\
\hline & Controls ( $N=27$ ) Mean (SD) & Patients ( $N=27$ ) Mean (SD) & \\
\hline Odour Threshold & $8.20(3.51)$ & $1.70(1.25)^{\mathrm{b}}$ & $2.20(1.50)$ \\
\hline Odour Discrimination & $12.78(1.19)$ & $7.74(3.37)^{\mathrm{b}}$ & $8.00(2.86)$ \\
\hline Odour Identification & $14.30(1.07)$ & $6.89(3.39)^{b}$ & $8.78(3.18)^{d}$ \\
\hline TDI & $34.36(3.59)$ & $16.23(6.32)^{\mathrm{b}}$ & $18.98(6.26)^{d}$ \\
\hline Left OB volume $\left(\mathrm{mm}^{3}\right)$ & $47.35(9.77)$ & $39.60(5.81)^{\mathrm{a}}$ & $40.64(5.97)^{c}$ \\
\hline Right OB volume $\left(\mathrm{mm}^{3}\right)$ & $50.41(7.21)$ & $40.43(7.45)^{\mathrm{b}}$ & $42.20(7.96)^{d}$ \\
\hline
\end{tabular}

TDI = sum of odour threshold, discrimination and identification scores. OB = olfactory bulb. OT = olfactory training. Superscript letters a and b indicate significant difference compared to controls, ${ }^{a} p<0.01,{ }^{b} p<0.0001$. Superscript letters $c$ and d reflect $p$-value of pairwise t-tests comparing preand post-OT means; ${ }^{c} p<0.05,{ }^{d} p<0.01$.

\section{Structure Image acquisition}

MRI scans were performed in Part 1 and 2 using a 3T GE scanner (Discovery MR-750, General Electric Healthcare, Chicago, IL, USA) with a 8-channel phase-array head coil (HDNV-Head). Full details are provided in the supplementary section.

\section{OB volume measurement}

A segmentation of each $\mathrm{OB}$ was performed using open source software 3D Slicer Version 4.10.2 to process T2-weighted images using previously described procedures ${ }^{(5)}$. OB volumes were obtained by manual bordering and following addition of all slices, which were multiplied by the slice thickness to yield volume.

\section{Olfactory training protocol}

In Part 2 of the study, participants completed a commonly used OT protocol, that is, smelling of four different odorants; rose, eucalyptus, lemon, and eugenol (cloves). Patients were instructed to smell each of the four odours individually, twice per day. The average duration of OT was 213 days, with a range of 181 days to 266 days.

\section{Results}

\section{Olfactory bulb volume}

A one-way ANOVA revealed controls had significantly larger left $(F(1,53)=12.53, p<0.001)$ and right $(F(1,53)=25.02, p<$ 0.001 ) OB volumes than patients (Table 1). After OT, patients had significantly larger left $(\mathrm{t}(26)=2.11, \mathrm{p}=0.045)$ and right $(\mathrm{t}(26)$ $=3.04, p=0.005$ ) OB volumes. An increase of $5.5 \mathrm{in} \mathrm{TDI} \mathrm{score} \mathrm{is}$ a clinically significant improvement in olfactory function and based on this benchmark, after OT, six of 27 patients (i.e., 22\%) demonstrated a clinically significant improvement.

\section{Olfactory function}

Patients performed significantly worse than controls on the three Sniffin' Sticks tests (Table 1). After OT, patients had signi- ficantly higher identification score $(t(26)=3.47, p=0.002)$, but the threshold score $(t(26)=1.81, p=0.082)$ and discrimination score $(t(26)=0.45, p=0.65)$, were not significant different after OT. However, patients' overall olfactory function score (i.e., TDI), significantly increased after OT $(\mathrm{t}(26)=3.45, \mathrm{p}=0.002)$.

\section{Discussion}

Consistent with previous findings, patients with idiopathic olfactory loss had significantly lower olfactory function and significantly smaller OB volumes ${ }^{(6)}$ compared to controls. Moreover, patients with idiopathic olfactory loss demonstrated a significant increase in overall olfactory function score after six months of OT, congruent with earlier research ${ }^{(4)}$. After OT, the idiopathic patients demonstrated a significant increase in $\mathrm{OB}$ volumes; a finding that is inconsistent to previous studies with TBI- and URTI-olfactory loss patients who evidenced no increase in OB volume ${ }^{(7,8)}$.

Reasons as to why only some types of patients with olfactory loss experience an increase in OB volume after OT may be due to the cause of the olfactory loss; with post-infectious patients particularly responsive to $\mathrm{OT}^{(4)}$. However, TBI patients with olfactory loss may have reduced neural plasticity due the nature of damage, such as axonal shearing and olfactory cleft obstruction. Methodological differences between OT studies may also explain why some olfactory loss patients do not evidence an increase of $\mathrm{OB}$ volume and others do, such as the use of different brain scanning equipment, volume measurement techniques, and software for $\mathrm{OB}$ volumetric measurement.

\section{Authorship contribution}

MKM: analysis, manuscript preparation and revisions. MM: data collection and analysis. PF: data collection and analysis. NA: data collection and analysis. TH: data collection, analysis and project management. 


\section{Conflict of interest}

The authors have no conflict of interests.

\section{Acknowledgement}

None.

\section{References}

1. Croy I, Nordin S, Hummel T. Olfactory disorders and quality of life-an updated Review. Chem Sens. 2014 39(3):185-194.

2. Sorokowska A, Drechsler E, K; arwowski M, Hummel T. Effects of Olfactory training: a meta-analysis. Rhinology. 2017 55:17-26.

3. Huart C, Rombaux P, Hummel T. Plasticity of the Human Olfactory System: The Olfactory Bulb. Molecules. 2013 18(9):11586-11600.

4. Seubert J, Freiherr J, Frasnelli J, Hummel T, Lundstrom JN Orbitofrontal cortex and olfactory bulb volume predict distinct aspects of olfactory performance in healthy subjects. Cereb Cortex. 2013 23:2448-2456.

5. Rombaux P. H, Duprez T, Hummel T. Olfactory bulb volume in the clinical assess ment of olfactory dysfunction. Rhinology. 2009 47(1):3-9.

6. Rombaux P, Potier H, Markessis E, Duprez T, Hummel T. Olfactory bulb volume and depth of olfactory sulcus in patients with idiopathic olfactory loss. Eur Arch OtoRhino-Laryngol. 2010 267(10):1551-1556.

7. Gellrich J, Han P, Manesse C, Betz A, Junghanns A, Raue C, Schriever VA, Hummel T. Brain volume changes in hyposmic patients before and after olfactory training. Laryngoscope. 2018 128:15311536.

8. Langdon C, Lehrer E, Berenguer J, Laxe S, Alobid I, Quintó L et al. Olfactory Training in Post-Traumatic Smell Impairment: Mild Improvement in Threshold Performances:
Results from a Randomized Controlled Trial. Journal of Neurotrauma. 2018 35:26412652.

\section{Mehmet K. Mahmut}

Smell and Taste Clinic

Department of Otorhinolaryngology

TU Dresden

Dresden

Germany

E-mail : mem.mahmut@mq.edu.au

\section{SUPPLEMENTARY INFORMATION}

\section{Participants}

Of the 27 patients with idiopathic olfactory loss, two reported phantosmia, two reported parosmia, one reported both phantosmia and parosmia and one patient reported dysgeusia. Two patients were current smokers. The reported mean duration of smell loss was 54 months (SD = 55). Only four controls were current smokers.

\section{Olfactory function: self-assessments}

To determine each participant's self-assessment of olfactory function after OT, in Part 2, they were asked three questions upon returning for the second MRI: 1) Did your olfactory function improve after OT? (worse, same or better?); 2) Did your parosmia symptoms improve after OT? (worse, same, better or gone/cured?); 3) Did your phantosmia symptoms improve after OT? (worse, same, better and gone/cured?).

Self-assessment of changes in olfactory ability, parosmia and phantosmia

Based on patients' qualitative, self-assessments regarding the impact of the OT: $56 \%$ patients indicated there was no change, $41 \%$ indicated it had improved and 3\% said it has worsened. In terms of the two patients who reported experiences of phantosmia before OT: one indicated there was no difference in their symptoms and one indicated their symptoms disappeared after OT. In terms of the two patients who reported experiences of parosmia before OT, both indicated there was no difference in their symptoms after OT. Finally, the one patient that reported experiences of both phantosmia and parosmia before OT, reported no change in either phantosmia or parosmia symptoms after OT.
Structure image acquisition

MRI scans were performed in Part 1 and 2 using a 3T GE scanner (Discovery MR-750, General Electric Healthcare, Chicago, IL, USA) with a 8-channel phase-array head coil (HDNV-Head). Using a 3D magnetization prepared isotropic fast spoiled gradient inversion recovery (FSPGR IR iso) sequence the T1-weighted images were acquired, with parameters: TR 7900 ms; TE 3.1 ms; TI 450 ms; FOV 240 mm; voxel size 1×1×1 mm; flip angle $12^{\circ}, 152$ contiguous slices of $1 \mathrm{~mm}$ thickness. Images were acquired in the sagittal plane oriented parallel to the cerebral falx. From this dataset axial and coronal images were reformatted with identical voxel size. OB-sequence included acquisition of 2-mm-thick T2-weighted fast spin-echo images, with $2 \mathrm{~mm} \times 2 \mathrm{~mm}$ pixel dimension, without inter-slice gap in the coronal plane covering the anterior and middle segments of skull base.

\section{Statistical analysis}

Data analysis was performed using SPSS (version 25, SPSS Inc., Chicago, IL, USA). The patients and controls were compared on the means scores on each of the six dependent variables using a one-way analysis of variance (ANOVA). There were no significant differences between controls and patients in terms of age, $F(1,69)<1, p=0.81$. The alpha level was set at $p=0.05$ for all statistical tests. A Bonferroni correction was used for post-hoc tests to control for Type-1 errors. 